\title{
Effects of yoga in men with prostate cancer on quality of life and immune response: a pilot randomized controlled trial
}

Dharam Kaushik (iD) ${ }^{1,2}$, Pankil K. Shah ${ }^{1}$, Neelam Mukherjee ${ }^{1}$, Niannian Ji ${ }^{1}$, Furkan Dursun ${ }^{1,2}$, Addanki P. Kumar ${ }^{2,3}$, lan M. ThompsonJr ${ }^{4}$, Ahmed M. Mansour ${ }^{1,2}$, Richapriya Jha ${ }^{1}$, Xiaoyu Yang ${ }^{3}$, Hanzhang Wang ${ }^{1}$, Nydia Darby ${ }^{5}$, J. Ricardo Rivero ${ }^{1}$, Robert S. Svatek ${ }^{1,2}$ and Michael A. Liss (D) ${ }^{1,2}$

(c) The Author(s) 2021

BACKGROUND: Diagnosis and treatment of prostate cancer is associated with anxiety, fear, and depression in up to one-third of men. Yoga improves health-related quality of life $(\mathrm{Q} o \mathrm{~L})$ in patients with several types of cancer, but evidence of its efficacy in enhancing QoL is lacking in prostate cancer.

METHODS: In this randomized controlled study, 29 men newly diagnosed with localized prostate cancer were randomized to yoga for 6 weeks $(n=14)$ or standard-of-care $(n=15)$ before radical prostatectomy. The primary outcome was self-reported QoL, assessed by the Expanded Prostate Index Composite (EPIC), Functional Assessment of Cancer Therapy-Prostate (FACT-P), Functional Assessment of Chronic Illness Therapy-Fatigue (FACIT-F), Functional Assessment of Cancer Therapy-General (FACT-G) at baseline, preoperatively, and 6 weeks postoperatively. Secondary outcomes were changes in immune cell status and cytokine levels with yoga.

RESULTS: The greatest benefit of yoga on QoL was seen in EPIC-sexual (mean difference, 8.5 points), FACIT-F (6.3 points), FACTFunctional wellbeing (8.6 points), FACT-physical wellbeing (5.5 points), and FACT-Social wellbeing (14.6 points). The yoga group showed increased numbers of circulating CD4+ and CD8 + T-cells, more production of interferon-gamma by natural killer cells, and increased Fc receptor III expression in natural killer cells. The yoga group also showed decreased numbers of regulatory T-cells, myeloid-derived suppressor cells, indicating antitumor activity, and reduction in inflammatory cytokine levels (granulocyte colonystimulating factor [0.55 (0.05-1.05), $p=0.03]$, monocyte chemoattractant protein [0.22 $(0.01-0.43), p=0.04]$, and FMS-like tyrosine kinase-3 ligand [0.91 $(-0.01,1.82), p=0.053]$.

CONCLUSIONS: Perioperative yoga exercise improved QoL, promoted an immune response, and attenuated inflammation in men with prostate cancer. Yoga is feasible in this setting and has benefits that require further investigation.

TRIAL REGISTRATION: clinicaltrials.org (NCT02620033).

Prostate Cancer and Prostatic Diseases (2022) 25:531-538; https://doi.org/10.1038/s41391-021-00470-w

\section{INTRODUCTION}

In 2020, there were 191,930 new prostate cancer cases in the US [1]. A diagnosis of prostate cancer may have profound psychological effects that contribute to poor physical, emotional, and social quality of life (QoL). Up to $30 \%$ of men diagnosed with prostate cancer experience significant anxiety, fear, and distress concerning the disease and treatment-related complications [2]. Moreover, the risk of suicide is doubled in the year following a diagnosis of prostate cancer [3]. Increasing use of natural approaches such as acupuncture, herbal supplements and vitamins, yoga, meditation, massage therapy and aromatherapy, coupled with the unmet need for effective management of QoL-related symptoms, has created a demand for integrative medicine in these men [4-6].

Many studies have demonstrated that yoga improves healthrelated QoL and emotional, physical, and mental wellbeing in patients with cancer. Mindfulness defined as "paying attention in a particular way, on purpose, in the present moment, and nonjudgmentally"-has been shown to be improved with yoga practice with focus on breath work $[7,8]$. Moreover, in addition to improving fitness, flexibility, and muscle tone, yoga lessens anxiety and stress [9-16].

There is also evidence indicating that yoga attenuates oxidative stress and chronic inflammation associated with stressful situations $[11,13,17]$. However, our understanding of the molecular mechanisms involved in these effects remains limited. Furthermore, most of the relevant studies have been performed in breast cancer, and there are limited data in prostate cancer. The aims of this pilot study were to assess the effects of a perioperative yoga exercise program on QoL, fatigue, sexual and urinary function, and mindfulness and

\footnotetext{
${ }^{1}$ Department of Urology, University of Texas Health San Antonio, San Antonio, TX, USA. ${ }^{2}$ Mays Cancer Center, University of Texas Health San Antonio, MD Anderson Cancer Center, San Antonio, TX, USA. ${ }^{3}$ Department of Molecular Medicine, University of Texas Health San Antonio, San Antonio, TX, USA. ${ }^{4}$ Christus Santa Rosa Health System, San Antonio, TX, USA. ${ }^{5}$ Nydia Yoga Therapy Studio, San Antonio, TX, USA. ${ }^{\times}$email: dkuro@me.com
} 
on the cellular immune response and proinflammatory marker levels.

\section{MATERIALS AND METHODS \\ Study design and participants}

This block randomized, open-label, parallel-group clinical trial included 29 men with prostate cancer who were scheduled for radical prostatectomy. This was a pilot study of a yoga program in men with prostate cancer to elicit a hypothesis and estimate an appropriate effect size. Patients were accrued from September 25, 2015, to February 6, 2019. The study inclusion criteria were as follows: age 30-80 years; pathologically and/or radiographically confirmed new diagnosis of localized prostate cancer; scheduled for radical prostatectomy (robotic-assisted or open); no active synchronous malignancy; not currently practicing yoga and/or meditation; adequate pain control; no neurological or musculoskeletal comorbidity that would interfere with exercise; willingness to be randomized to either study group and undergo phlebotomy; and ability to provide informed consent. Patients with an absolute contraindication to exercise testing or a psychotic, addiction-related, or major cognitive disorder were excluded. The patients were randomized into a yoga group $(n=14)$ that participated in a yoga program for 6 weeks preoperatively and postoperatively and a control group $(n=15)$ that received standard-of-care only. Control group were patients with new diagnosis of prostate cancer who did not undergo yoga intervention prior to their surgery. All patients completed healthrelated QoL surveys at baseline (6 weeks preoperatively), immediately before surgery, and 6 weeks postoperatively. Blood samples were collected at these three time points for examination of immune cell status and cytokine levels. The full study protocol is provided as Supplementary Text 1.

The study was approved by the institutional review board of Long School of Medicine, UT Health San Antonio (approval: HSC20150406H). Written informed consent was obtained from all study participants.

\section{Yoga program}

The yoga intervention was developed for patients with prostate cancer as a collaborative effort between the Thrivewell Cancer Foundation, a local yoga studio, and the lead author (DK). The program consisted of $60 \mathrm{~min}$ of yoga exercise twice weekly for 6 weeks preoperatively (depending on surgeon and theater availability) and for 6 weeks starting 3-6 weeks postoperatively. The yoga sessions were led by certified instructors from the ThriveWell Cancer Foundation and the local yoga studio. Sessions were held at various locations in San Antonio, TX, and participants could choose their most convenient location. We utilized Hatha yoga method-Hatha yoga generally refers to the practice's focus on use of physical postures. Hatha yoga was combined with focused attention on gentle breath while moving with awareness through the practice to gently mobilize major joint in the body. The study practice also provided the breathing and pelvic floor engagement awareness in seated meditation at the beginning of yoga practice. Each participant was shown how to perform yoga correctly and safely, with tailoring of exercises to their comfort level. The instructors monitored patients' progress by observing their ability to breathe smoothly, rhythmically, and continuously while performing yoga. The study was performed in San Antonio, Texas.

\section{Clinical outcomes}

Health-related QoL was assessed using the Functional Assessment of Cancer Therapy-Prostate (FACT-P) scale. FACT-P is a modification of the FACT scale, a 27-item measure of QoL across the domains of physical, social/family, emotional, and functional wellbeing, and contains 12 additional items specific to the impact of prostate cancer symptoms. Therefore, the FACT-P yields both a prostate cancer-related QoL score and a total QoL score [18]. The Five Facets of Mindfulness Questionnaire was used to evaluate the effects of yoga on everyday mindfulness. This 39-item measure includes five domains (Observe, Describe, Act with Awareness, Non-judging of Inner Experience, and Nonreactivity to Inner Experience) and has been validated in both English and Spanish [19].

Cancer-specific fatigue was measured using the 13-item Functional Assessment of Chronic Illness Therapy-Fatigue (FACIT-F) questionnaire [20], urinary continence using the 7-item Expanded Prostate Index Composite (EPIC) urinary questionnaire [21], and erectile function using the 9-item EPIC-sexual function questionnaire [22]. All three questionnaires have been confirmed to have good reliability and validity.

\section{Analysis of immune cells}

Cryopreserved human peripheral blood mononuclear cells (PBMCs) were obtained at all assessment times and processed for immune analysis as previously described [23].

Briefly, PBMC cryovials were thawed rapidly at $37^{\circ} \mathrm{C}$ and washed in $9 \mathrm{ml}$ of warm serum-free RPMI-1640 medium (Corning, Corning, New York, NY). Single-cell PBMC suspensions were quantified for total number of live cells using an automated cell counter (Vi-Cell XR, Beckman Coulter, Brea, CA). After centrifugation at $4^{\circ} \mathrm{C}$ and $1200 \mathrm{rpm}$ for $5 \mathrm{~min}$, the cell pellets were resuspended with ice-cold flow buffer (sterile $2 \%$ fetal bovine serum in phosphate-buffered saline) at a maximum of $1 \times 10^{6}$ cells with a staining volume of $100 \mu \mathrm{l}$ on 96-well U-bottom plates; they were then incubated with human Fc blocker for 20 min and stained using fixable viability dye and fluorochrome-conjugated anti-human monoclonal antibodies for $45 \mathrm{~min}$ at $4^{\circ} \mathrm{C}$ in the dark (Supplementary Table 1). For examination of the cytokine response in immune cells, the thawed PBMCs were resuspended in complete RPMI-1640 medium containing 10\% fetal bovine serum, penicillin/streptomycin, and L-glutamine (Corning) on a 96-well Ubottom plate and incubated in a resting state at $37^{\circ} \mathrm{C}$ overnight. Next, the cells were stimulated for $5 \mathrm{~h}$ using Cell Activation Cocktail (BioLegend, San Diego, CA) at a dilution of 1:500. Cell surface and intracellular staining was then performed using a Fixation/Permeabilization Solution Kit (Cytofix/ Cytoperm, BD Biosciences, San Jose, CA). The stained samples were analyzed using an LSRII cytometer (BD Biosciences) with FACS Diva software. Using the gating strategy for immune cell subsets, all live PBMCs were first gated from singlets and fixable viability dye-negative populations. $\mathrm{CD}^{+}$and $\mathrm{CD}^{+}{ }^{+}$-cells were gated under a live $\mathrm{CD}^{+}$population. Regulatory T-cells were gated as live $\mathrm{CD}^{+}{ }^{\mathrm{CD}} 4^{+} \mathrm{CD} 25^{+} \mathrm{Foxp}^{+}$cells and $\gamma \delta$ T-cells were gated as live $\mathrm{CD}^{+} \mathrm{CD}^{-} \mathrm{CD}^{-}$cells. Natural killer (NK) cells were gated as a live $\mathrm{CD}^{-}{ }^{-} \mathrm{CD} 56^{+}$population and subdivided further into CD56 ${ }^{\text {bright }}$ and CD56 ${ }^{\text {dim }}$ NK cells. Myeloid populations were identified as live $C D 11 b^{+}$cells. Myeloid cells were further gated into subpopulations as follows: macrophages as live $\mathrm{CD} 11 \mathrm{~b}^{+} \mathrm{CD}^{+} 8^{+}$cells were divided further into $\mathrm{M} 1$ macrophages (live $\mathrm{CD} 11 \mathrm{~b}^{+} \mathrm{CD} 68^{+} \mathrm{CD} 64^{+} \mathrm{CD} 80^{+}$cells) and $\mathrm{M} 2$ macrophages (live $\mathrm{CD} 11 \mathrm{~b}^{+} \mathrm{CD} 68^{+} \mathrm{CD} 23^{+} \mathrm{CD} 163^{+}$cells), MDSCs as live $\mathrm{CD} 11 \mathrm{~b}^{+}$ $\mathrm{CD}_{3}{ }^{+}$cells further subgated into M-MDSCs (live $\mathrm{CD} 11 \mathrm{~b}^{+} \mathrm{CD} 33^{+}$ $\mathrm{HLADR}^{-} \mathrm{CD} 15^{-} \mathrm{CD} 14^{+}$cells) and G-MDSCs (live $\mathrm{CD} 11 \mathrm{~b}^{+} \mathrm{CD} 33^{+} \mathrm{CD} 15^{+}$ $\mathrm{CD} 14^{-}$cells), neutrophils as live $\mathrm{CD} 11 \mathrm{~b}^{+} \mathrm{CD} 15^{+} \mathrm{CD} 14^{-}$cells, and dendritic cells as live $\mathrm{CD} 11 \mathrm{~b}^{+} \mathrm{CD} 14^{+} \mathrm{CD} 15^{-}$cells. The gating strategy for expression of subsequent cell surface or intracellular markers on each immune cell subset was based on a fluorescence minus one control for each individual marker/monoclonal antibody.

\section{Evaluation of cytokines}

Plasma samples from the 15 patients in the control group and 14 patients in the yoga group were analyzed at the Bioanalytics and Single-Cell Core Laboratory in the Department of Molecular Medicine at UT Health San Antonio. The samples were centrifuged at $10,000 \mathrm{~g}$ and $4{ }^{\circ} \mathrm{C}$ for $10 \mathrm{~min}$. Next, $25 \mu \mathrm{l}$ of the clarified top portion were collected and analyzed in duplicate using the FlexMap 3D platform system (Luminex, Austin, TX) with the Milliplex MAP human cytokine/chemokine Magnetic Bead Panel-Immunology Multiplex Assay kit (Cat \# HCYTMAG-60K-PX38; EMD Millipore, Billerica, MA). The following cytokines and chemokines were analyzed: epidermal growth factor, fibroblast growth factor-2, FMS-like tyrosine kinase (FIt)-3 ligand, fractalkine, granulocyte colony-stimulating factor (G-CSF), granulocytemacrophage colony-stimulating factor, growth-regulated protein, interferon (IFN)-a2, IFN- $\gamma$, interleukin (IL)-1 a, IL-1 $\beta$, IL-1ra, IL-2, IL-3, IL-4, IL-5, IL-6, IL-7, IL8, IL-9, IL-10, IL-12 (p40), IL-12 (p70), IL-13, IL-15, IL-17A, IFN-gamma-induced protein (IP)-10, monocyte chemoattractant protein (MCP)-1, MCP-3, macrophage-derived chemokine (CCL22), macrophage inflammatory protein- $1 \alpha / 1 \beta$, transforming growth factor- $a$, tumor necrosis factor (TNF)- $a$, TNF$\beta$, vascular endothelial growth factor, eotaxin/CCL11, and sCD40L.

\section{Statistical analysis}

Domain and subdomain scores for all patient-reported outcomes were calculated as per their respective guidelines and then scaled to a 0-100 score. A higher score indicates better health-related QoL. All continuous variables were evaluated for normality using the Kolmogorov-Smirnov test. Between-group differences in sociodemographic, clinical, and immune parameters were evaluated at baseline using the Student's $t$-test for continuous variables and the chi-squared test for categorical variables (Fisher's exact test was used as appropriate). The effect of the intervention was evaluated by calculating the improvement in 


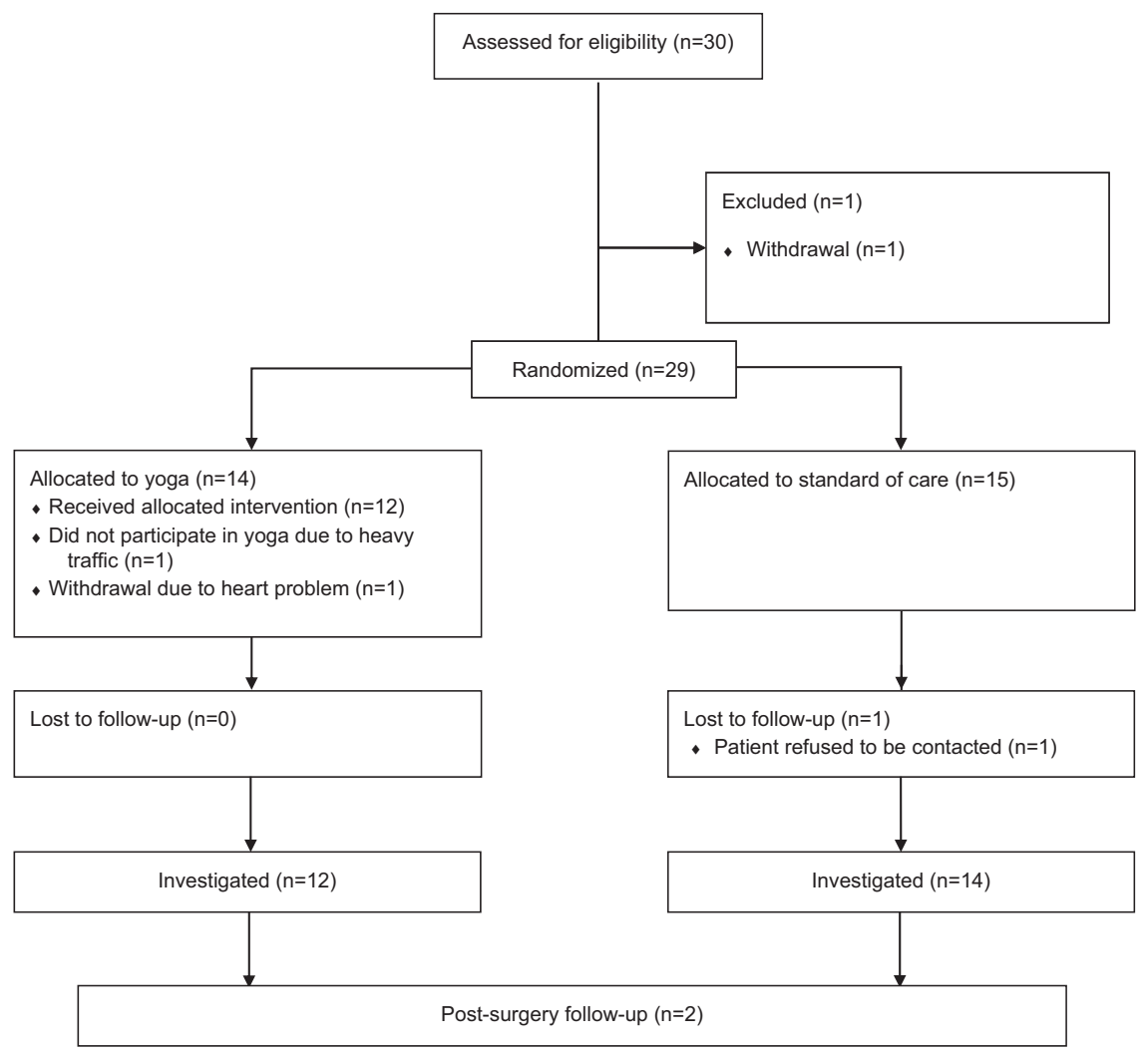

Fig. 1 CONSORT diagram. Flowchart of patient enrollment process for the study.

patient-reported outcome scores, cytokine expression, and numbers of immune cells between baseline and immediately before surgery. Betweengroup differences were evaluated using the Student's $t$-test if the variable was normally distributed and the Wilcoxon rank-sum test if not. The significance level was set to $a=0.05$ (two-tailed) but not adjusted for multiple comparisons using methods such as Bonferroni correction because we were not testing any a priori hypothesis. The primary outcome was self-reported QoL at baseline, preoperatively, and 6 weeks postoperatively. Secondary outcomes were changes in immune cell status and cytokine levels with yoga. The analysis was restricted to only the first two time points because only two of the 15 participants in the yoga group completed the third time point (per-protocol analysis). All statistical analyses were performed using $R$ software (R Foundation for Statistical Computing, Vienna, Austria).

\section{RESULTS}

Twenty-nine of 30 patients assessed for eligibility were randomized to the yoga group $(n=14)$ or the control group $(n=15)$. Twelve patients in the yoga group completed their yoga program and could be followed up before surgery and one in the control group was lost to follow-up. Therefore, complete data for 26 patients were available for the analyses (Fig. 1).

\section{Patient demographics and clinical characteristics}

The baseline sociodemographic and clinical characteristics are shown in Supplementary Table 2. The median patient age was 60 years (IQR 59-61) in the control group and 56 years (IQR 55-60.5) in the yoga group. Most of the patients had organ-confined disease. Approximately $23 \%$ of the cohort was Hispanic. There was no significant between-group difference in QoL at baseline (Supplementary Table 3).

\section{Clinical outcomes}

Table 1 shows the changes in mean scores in both study groups at the scale and subscale levels. Overall, there was a statistically nonsignificant trend towards improvement in sexual function (EPIC questionnaire, yoga vs control: 9.1 vs. $0.6 ; p=0.098$ ), fatigue (FACIT-F questionnaire, 1.8 vs. $-4.5 ; p=0.098)$, general QoL (FACT-P: 1.9 vs. $-6.3 ; p=0.065$ ), and prostate-specific QoL (0.6 vs. $-5.3 ; p=0.08)$. We then calculated the minimally important difference (MID), namely, the minimal effect that would be meaningful to patients, for each patient-reported QoL outcome by stratifying our data to one-third of a standard deviation. This amount of change in the standard deviation has been shown to have a clinically meaningful impact on a patient's QoL $[24,25]$. We then found that the FACT-P, FACT-General, FACIT-F, and EPICSexual scores were improved meaningfully by yoga (Fig. 2). On further substratification of these scales using the MID, there were improvements in the FACT-P physical, social, and functional wellbeing scores and in EPIC-Sexual function (Supplementary Fig. 1) in the yoga group.

\section{Immune cells}

We characterized lymphocytes from the patients' blood samples using multi-parametric gating flow cytometry (Supplementary Fig. 2 shows the results and Supplementary Table 1 lists the antibodies used). We then analyzed the immune cell data by creating a t-distributed stochastic neighbor embedding (tSNE) plot, which is a non-linear dimensionality reduction algorithm that provides "big picture" data. A global t-SNE map of PBMCs was obtained using a 12-parameter flow cytometry panel. Using the tSNE algorithm, we identified qualitative phenotypic differences between the yoga group and control group (Fig. 3a). We further delineated the populations of T-cells, NK cells, and subsets of myeloid cells and MDSCs. We then plotted the differences in frequencies and absolute numbers of immune cells between the study groups using box and whisker plots (Supplementary Fig. 3a-e). We identified an increased IFN- $\gamma$ response in peripheral cytotoxic CD4+ $(p=0.007)$ and CD8 $+(p=0.004)$ cells in the yoga group in comparison with the control group (Fig. 3b). Levels of 
Table 1. Improvement in patient-reported outcomes by study group at the scale and subscale levels.

\begin{tabular}{|c|c|c|c|c|c|c|}
\hline \multirow[t]{2}{*}{ Scale level } & \multirow[b]{2}{*}{ Domain } & \multicolumn{2}{|c|}{ Study group } & \multirow[b]{2}{*}{$P$-value } & \multirow[b]{2}{*}{$\begin{array}{l}\text { Effect size with } 95 \% \\
\text { confidence interval }\end{array}$} & \multirow[b]{2}{*}{$\begin{array}{l}\text { Greater } \\
\text { than MID }\end{array}$} \\
\hline & & $\begin{array}{l}\text { Yoga } \\
\text { mean (SE) }\end{array}$ & $\begin{array}{l}\text { Control } \\
\text { mean (SE) }\end{array}$ & & & \\
\hline \multicolumn{7}{|c|}{ Expanded prostate cancer index composite } \\
\hline & Sexual & $9.1(2.6)$ & $0.6(4.2)$ & 0.098 & $8.5(-1.7,18.8)$ & Yes \\
\hline & Urinary & $-0.2(2.7)$ & $1.4(1.9)$ & 0.638 & $-1.6(-8.4,5.3)$ & No \\
\hline \multicolumn{7}{|c|}{ Functional assessment of chronic illness therapy } \\
\hline & Trial outcome index & $1.8(2.5)$ & $-3.0(2.5)$ & 0.187 & $4.8(-2.5,12.1)$ & No \\
\hline & Fatigue & $1.8(2.3)$ & $-4.5(2.8)$ & 0.098 & $6.3(-1.3,13.8)$ & Yes \\
\hline \multicolumn{7}{|c|}{ Functional assessment of cancer therapy } \\
\hline & Trial outcome index & $0(2.5)$ & $-4.2(2.0)$ & 0.200 & $4.2(-2.4,10.8)$ & No \\
\hline & General & $1.9(2.7)$ & $-6.3(3.2)$ & 0.065 & $8.2(-0.6,16.9)$ & Yes \\
\hline & Prostate & $0.6(2.2)$ & $-5.3(2.5)$ & 0.086 & $5.9(-0.9,12.7)$ & Yes \\
\hline \multicolumn{2}{|c|}{ Five facet mindfulness questionnaire } & $-1.5(2.8)$ & $-0.6(1.2)$ & 0.783 & $-0.9(-7.4,5.7)$ & No \\
\hline \multicolumn{2}{|c|}{ Subscale level } & \multicolumn{2}{|c|}{ Study group } & & & \\
\hline \multicolumn{2}{|c|}{ Expanded prostate cancer index composite } & $\begin{array}{l}\text { Yoga } \\
\text { mean (SE) }\end{array}$ & $\begin{array}{l}\text { Control } \\
\text { mean (SE) }\end{array}$ & $P$-value & $\begin{array}{l}\text { Effect size with } 95 \% \\
\text { confidence interval }\end{array}$ & $\begin{array}{l}\text { Greater } \\
\text { than MID }\end{array}$ \\
\hline & Sexual bother & $11.5(4.7)$ & $3.8(7.0)$ & 0.376 & $7.6(-9.9,25.1)$ & No \\
\hline & Sexual function & $8.1(2.7)$ & $-0.9(3.2)$ & 0.043 & $9.0(0.3,17.6)$ & Yes \\
\hline & Urinary bother & $-0.9(3.3)$ & $1.1(2.5)$ & 0.635 & $-2.0(-10.6,6.6)$ & No \\
\hline & Urinary function & $-0.7(3.5)$ & $1.8(2.6)$ & 0.577 & $-2.5(-11.5,6.6)$ & No \\
\hline & Urinary incontinence & $-1.9(4.5)$ & $-2.6(2.3)$ & 0.894 & $0.7(-10,11.4)$ & No \\
\hline & Urinary obstructive & $0.1(3.0)$ & $4.1(2.4)$ & 0.308 & $-4.0(-12,4)$ & No \\
\hline \multicolumn{7}{|c|}{ Functional assessment of cancer therapy } \\
\hline & Emotional wellbeing & $-1.0(4.3)$ & $-4.2(3.4)$ & 0.573 & $3.1(-8.2,14.5)$ & No \\
\hline & Fatigue & $1.6(2.4)$ & $-0.7(2.8)$ & 0.536 & $2.3(-5.3,9.9)$ & No \\
\hline & Functional wellbeing & $1.2(4.4)$ & $-7.4(2.8)$ & 0.113 & $8.6(-2.2,19.5)$ & Yes \\
\hline & $\begin{array}{l}\text { Prostate cancer } \\
\text { subscale }\end{array}$ & $-2.3(2.7)$ & $-3(2.2)$ & 0.834 & $0.7(-6.4,7.9)$ & No \\
\hline & Physical wellbeing & $2.7(3.2)$ & $-2.8(3.2)$ & 0.241 & $5.5(-4,15)$ & Yes \\
\hline & Social wellbeing & $4.2(3.0)$ & $-10.4(7.9)$ & 0.103 & $14.6(-3.3,32.5)$ & Yes \\
\hline \multicolumn{7}{|c|}{ Five facet mindfulness questionnaire } \\
\hline & Awareness & $-2.6(4.2)$ & $1.7(3.3)$ & 0.429 & $-4.3(-15.3,6.8)$ & No \\
\hline & Describing & $-1.8(3.9)$ & $-4.1(2.4)$ & 0.629 & $2.3(-7.4,11.9)$ & No \\
\hline & Non-judging & $-8.9(3.2)$ & $-1.2(3.1)$ & 0.101 & $-7.7(-17,1.6)$ & No \\
\hline & Nonreactive & $5.7(4.3)$ & $3(2.9)$ & 0.619 & $2.6(-8.3,13.5)$ & No \\
\hline & Observing & $1.0(5.8)$ & $-2.2(4.1)$ & 0.656 & $3.2(-11.6,18)$ & No \\
\hline
\end{tabular}

MID minimally important difference, SE standard error.

regulatory T-cells $(p=0.029)$ and $\operatorname{MDSCs}(p=0.002)$ were reduced in the yoga group when compared with the control group, indicating enhanced antitumor activity in patients who practiced yoga. We also identified increased Fc receptor III $(p=0.041)$ and IFN- $\gamma(p=0.026)$ expression in NK cells, indicating a robust immune response as well as antitumor activity.

\section{Cytokines}

A statistically significant reduction in expression of inflammatory cytokines was identified in the yoga group, namely, G-CSF $(0.55$ [0.05-1.05]; $p=0.032)$, MCP-1 (0.22 [0.01-0.43]; $p=0.044)$, and Flt3 ligand (0.91 [-0.01, 1.82]; $p=0.053$ ) (Fig. 4). Changes in levels of 38 cytokines from baseline are compared between the study groups in Supplementary Fig. 4 and Supplementary Table 4.

\section{DISCUSSION}

Although there have been some small retrospective studies and one relevant randomized trial [14], the effects of yoga in patients with prostate cancer remain unclear, particularly regarding QoL and its molecular impact. Therefore, we designed this clinical trial to obtain preliminary clinical and translational data.

Our preliminary data add to the literature by providing a molecular explanation for the similar improvements in QoL seen in patients with prostate cancer. The greatest impact of yoga was on sexual function, fatigue, prostate cancer-specific QoL, and physical, social, and functional wellbeing. Our data also indicate that yoga modulates several key immune cells that are important drivers of antitumor activity. Furthermore, the analysis of cytokines/chemokines suggests that yoga attenuates the inflammatory response.

Our data demonstrate a positive effect of yoga in several clinical domains. First, we observed a significant improvement in QoL in the perioperative setting, which was reflected in enhanced physical, social, and functional wellbeing as well as improvement in symptoms of fatigue and stress. These findings are consistent with those of a trial by Ben-Josef et al. [14] in which 50 patients with prostate cancer undergoing radiation therapy were allocated to yoga classes $(n=22)$ or standard-of-care $(n=28)$ for $6-9$ weeks. In that study, patients in the yoga group experienced significantly less global fatigue and severity of fatigue than those in the control 


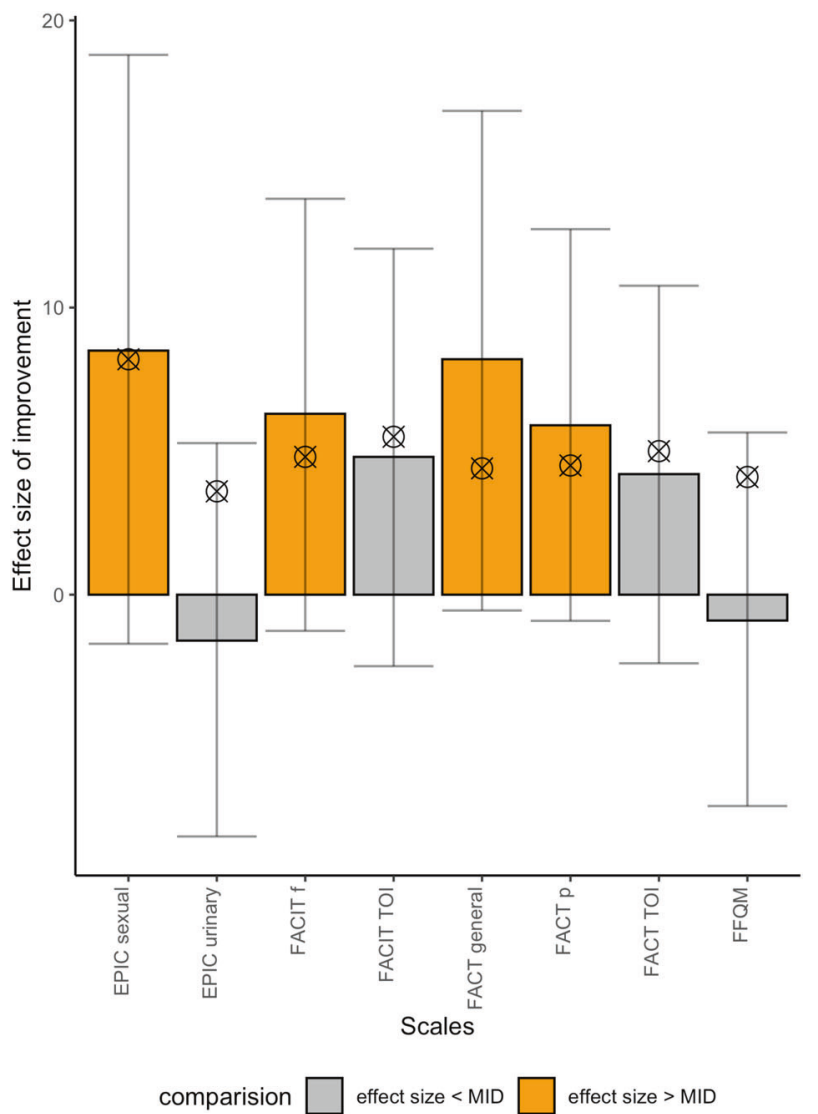

Fig. 2 Effect size of the yoga intervention compared to the minimally important difference (MID) with $95 \%$ confidence interval. The effect size was calculated as the difference between the postintervention score minus the preintervention score in the yoga group and the postintervention score minus the preintervention score in the control group. The MID was calculated as one-third of the overall standard deviation of the patient-reported scale item at baseline. The $\otimes$ mark on the graph indicates the MID for the scale item. EPIC Expanded Prostate Index Composite, FACIT Functional Assessment of Chronic Illness Therapy, FACIT-F FACIT-Fatigue, FACITG FACIT-General, FACT Functional Assessment of Chronic Illness Therapy, FFOM Five Facets of Mindfulness, TOI Trial Outcome Index.

group ( $p<0.0001)$. Moreover, as in our study, sexual health scores were better in their yoga group. Overall, our data and those of Ben-Josef et al. demonstrate that yoga has a positive effect on emotional, physical, and social scores.

Perioperative exercise studies for patients who underwent radical prostatectomy are mostly focused on the urinary continence after the surgery. Few studies with various perioperative exercise interventions studied the impact of exercise on cancer-specific QoL. In their randomized control trial assessing 49 patients, Park et al. [26] reported that a postoperative combined exercise intervention results in improvement of physical function and QoL. In a large systematic review and meta-analyses of 1057 prostate cancer patients enrolled in 13 randomized clinical trials, exercise intervention significantly improved fatigue symptoms [mean difference (MD) 4.83, 95\% Cl 3.24-6.43; $p<0.00001$ ] as assessed according to the Functional Assessment of Cancer Therapy (FACT)-Fatigue scale. Fatigue remained improved at 6 months (MD 3.60, 95\% Cl 2.80-5.12; $p<0.00001$ ). Furthermore, exercise interventions improved QOL measured using the FACTGeneral (MD 3.93, 95\% Cl 1.37-5.92; $p=0.003$ ) and FACT-Prostate (MD 3.85, 95\% Cl 1.25-6.46; $p=0.04$ ) scales [27].

A recent multi-institutional study examined the effect of yoga on quality of sleep in 410 cancer survivors (>90\% female) who a Control Group

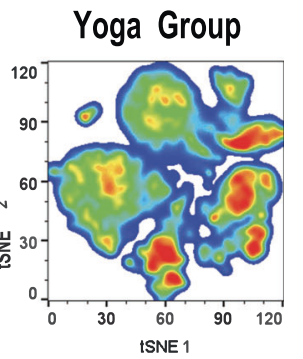

b

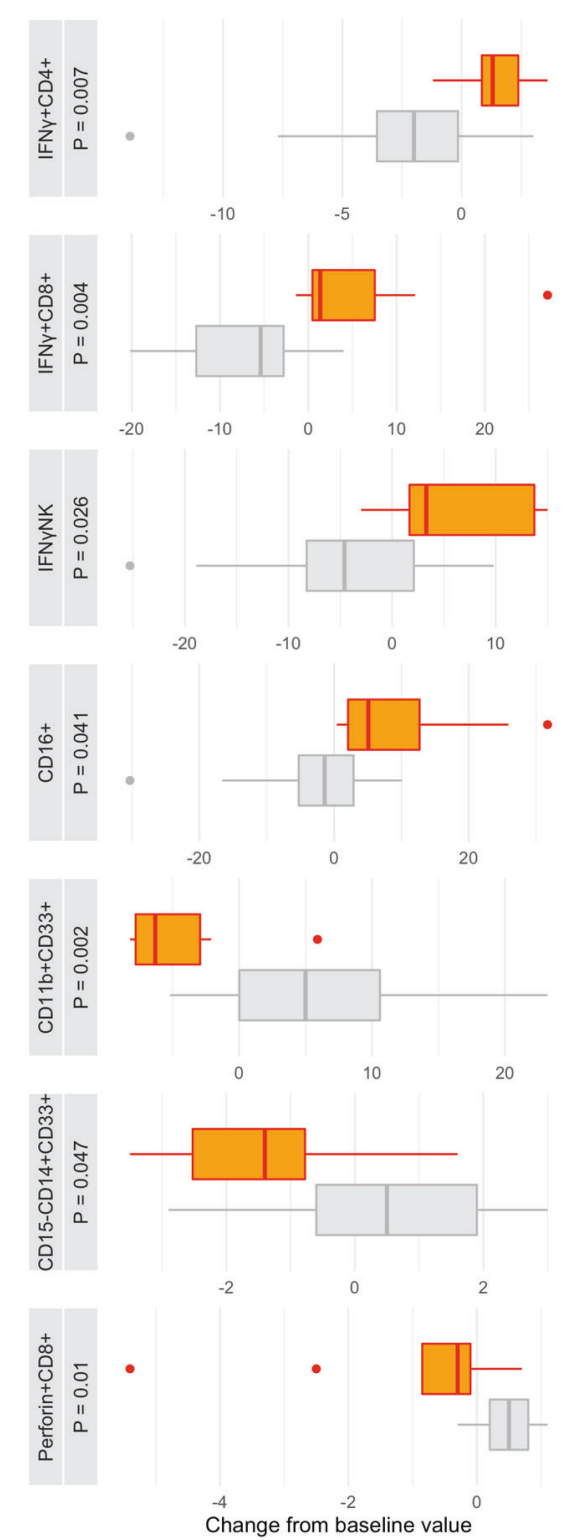

Study Group

Control Group

官 Yoga Group

Fig. 3 Analysis of immune cell data. a A t-distributed stochastic neighbor embedding (tSNE map) showing differences in immune cell phenotypes between the yoga group and the control group. b Box and whiskers plot of immune markers (IFN $\gamma+\mathrm{CD} 4+$, IFN $\gamma+$ CD8+, IFN $\gamma$ NK, CD16+, CD11b+CD33+, CD15-CD14+CD33+, perforin $+C D 8+$ ) between samples obtained from 13 patients in the control group and those obtained from 6 patients in the yoga group at endpoint. 


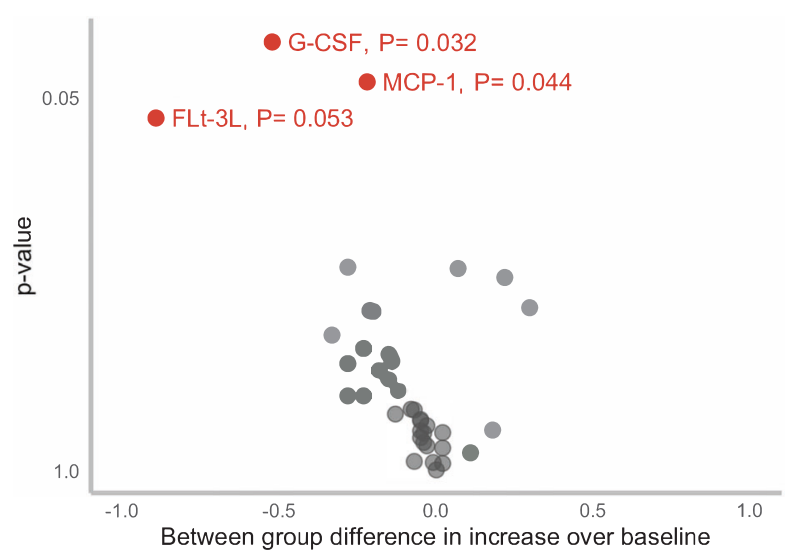

Fig. 4 Analysis of cytokine data. Volcano plot showing the difference in increase in expression levels of granulocyte colonystimulating factor (G-CSF), monocyte chemoattractant protein (MCP-1) and FMS-like tyrosine kinase-3 ligand (Flt-3 ligand) from baseline between the yoga group and the control group granulocyte colony-stimulating factor [0.55 $(0.05-1.05), p=0.03]$, monocyte chemoattractant protein [0.22 $(0.01-0.43), p=0.04]$, and FMS-like tyrosine kinase-3 ligand.

were randomly assigned to 4 weeks of yoga $(n=206)$ or standardof-care $(n=204)$ [9]. Compared with the control group, the yoga group demonstrated significant global improvements in quality, duration, and efficiency of sleep and less use of sleep medication. Longer-term follow-up of that cohort revealed significant improvement in all subdomains of cancer-related fatigue [28].

The Society for Integrative Oncology has produced an evidencebased guideline on use of integrative therapies during and after treatment of breast cancer that has been endorsed by an American Society of Clinical Oncology expert panel [29]. This guideline recommends yoga for reduction of anxiety and stress, amelioration of depression/mood, and improvement of QoL. Our data add to the literature by providing a molecular explanation for the similar improvements in QoL seen in patients with prostate cancer.

There are data suggesting that psychological stress has a negative effect on the adaptive cellular immune response, including decreased production of NK cells and T-cells. Chronic stress results in stimulation of the hypothalamic-pituitary-adrenal axis, which produces glucocorticoids, and the sympathetic-adrenal axis, which produces catecholamines [30]. Leukocytes have receptors for these stress-related hormones and can modulate their binding [31]. T-cells have more of these receptors and are exquisitely sensitive to fluctuations in stress hormone levels. Recent data show that stress and anxiety may lead to metabolic changes that impair the function of CD4+ T-cells [32]. In the present study, we identified a robust IFN- $\gamma$ response in CD4+ and CD8 + cells, increased expression of the Fc receptor (CD16) in NK cells, and decreases in numbers of regulatory T-cells and MDSCs. These findings, although only hypothesis-generating, point to a strong immune response, less stress, and better QoL in patients with prostate cancer who practice yoga. Future studies are needed to clarify the impact of yoga on T-cell subpopulations.

Research has shown a relationship between persistent fatigue and overactivation of the inflammatory network in patients with cancer. Other studies have shown an association between QoL indicators, including fatigue, anxiety, and depression levels, and increased production of proinflammatory cytokines, including IL- 6 and TNF-a [33]. Our data show that 6 weeks of a yoga exercise program reduced fatigue and expression of proinflammatory markers, including G-CSF, MCP-1, and Flt-3 ligand. G-CSF has been shown to activate production of endothelial cells and cytokines and to promote angiogenesis [34]. MCP-1 is a chemokine that is expressed by glial cells and neurons. Higher plasma MCP-1 levels are associated with more rapid and severe cognitive decline secondary to neuronal loss whereas lower levels are neuroprotective [35, 36]. There is emerging data showing that MCP-1 acts as a potent chemotactic factor regulating stromalepithelial cell in prostate cancer. It regulates prostate cancer motility and proliferation at the site of the bone microenvironment and overexpression of its receptors (CCR2 and CCRL1) may contribute to the progression and biochemical failure of prostate cancer [37]. Finally, higher expression levels of Flt-3 ligand have been linked to an autoimmune response and to chronic inflammatory responses in the lung, central nervous system, and gastrointestinal tract [38]. By lessening inflammation and fatigue and improving mood, yoga is an ideal exercise that can be modified for individuals with a sedentary lifestyle or functional limitations [11, 16, 28, 39-41]. Our pilot data are consistent with those of previous retrospective studies demonstrating the beneficial effects of yoga on several psychological and QoL outcomes, add granularity at the molecular level, and identify putative inflammatory markers for future research.

This study has several limitations. First, the study cohort was small. Second, assessments were performed at only two time points and adherence rates are not available. Third, given that we were not testing any a priori hypothesis, the significance level was not adjusted for multiple comparisons using methods such as Bonferroni correction. Therefore, our data is hypothesis-generating and further research is required.

In conclusion, our present findings indicate that yoga improves QoL, generates a robust immune response, and attenuates expression of key inflammatory cytokines in men with newly diagnosed prostate cancer. Our data shows that patients are motivated to perform yoga in the preoperative setting (no attrition) but not in the postoperative period (high attrition). Larger-scale studies are needed to replicate these results. Future studies should incorporate a translational component to clarify the mechanism via which yoga improves QoL and examine the effects of yoga on progression and recurrence of prostate cancer.

\section{DATA AVAILABILITY}

The principal investigator (DK) and biostatistician (PS) had full access to all the data in the study and takes responsibility for the integrity of the data and the accuracy of the data analysis.

\section{REFERENCES}

1. Siegel RL, Miller KD, Jemal A. Cancer statistics, 2020. CA Cancer J Clin. 2020;70:7-30. 2. Sanda MG, Dunn RL, Michalski J, Sandler HM, Northouse L, Hembroff L, et al. Quality of life and satisfaction with outcome among prostate-cancer survivors. N. Engl J Med. 2008;358:1250-61.

3. Guo Z, Gan S, Li Y, Gu C, Xiang S, Zhou J, et al. Incidence and risk factors of suicide after a prostate cancer diagnosis: a meta-analysis of observational studies. Prostate Cancer Prostatic Dis. 2018;21:499-508.

4. Wolf CPJG, Rachow T, Ernst T, Hochhaus A, Zomorodbakhsch B, Foller S, et al. Complementary and alternative medicine (CAM) supplements in cancer outpatients: analyses of usage and of interaction risks with cancer treatment. J Cancer Res Clin Oncol. 2021. https://doi.org/10.1007/s00432-021-03675-7.

5. Greenlee H, Neugut Al, Falci L, Hillyer GC, Buono D, Mandelblatt JS, et al. Association between Complementary and Alternative Medicine Use and Breast Cancer Chemotherapy Initiation the Breast Cancer Quality of Care (BQUAL) Study. JAMA Oncol. 2016;2:1170-6.

6. Richardson MA, Sanders T, Palmer JL, Greisinger A, Singletary SE. Complementary/alternative medicine use in a comprehensive cancer center and the implications for oncology. J Clin Oncol. 2000;18:2505-14.

7. Kabat-Zinn J. Mindfulness-based interventions in context: past, present, and future. Clin Psychol Sci Pr. 2003;10:144-56.

8. Gaiswinkler L, Unterrainer HF. The relationship between yoga involvement, mindfulness and psychological well-being. Complement Ther Med. 2016;26:123-7.

9. Mustian KM, Sprod LK, Janelsins M, Peppone LJ, Palesh OG, Chandwani K, et al. Multicenter, randomized controlled trial of yoga for sleep quality among cancer survivors. J Clin Oncol. 2013;31:3233-41.

10. Chandwani KD, Perkins G, Nagendra HR, Raghuram NV, Spelman A, Nagarathna R, et al. Randomized, controlled trial of yoga in women with breast cancer undergoing radiotherapy. J Clin Oncol. 2014;32:1058-65. 
11. Kiecolt-Glaser JK, Bennett JM, Andridge R, Peng J, Shapiro CL, Malarkey WB, et al. Yoga's impact on inflammation, mood, and fatigue in breast cancer survivors: A randomized controlled trial. J Clin Oncol. 2014;32:1040-9.

12. Taso CJ, Lin HS, Lin WL, Chen SM, Huang WT, Chen SW. The effect of yoga exercise on improving depression, anxiety, and fatigue in women with breast cancer: A randomized controlled trial. J Nurs Res. 2014;22:155-64.

13. Harkess KN, Ryan J, Delfabbro PH, Cohen-Woods S. Preliminary indications of the effect of a brief yoga intervention on markers of inflammation and DNA methylation in chronically stressed women. Transl Psychiatry. 2016;6. https://doi. org/10.1038/tp.2016.234.

14. Ben-Josef AM, Chen J, Wileyto P, Doucette A, Bekelman J, Christodouleas J, et al. Effect of Eischens Yoga During Radiation Therapy on Prostate Cancer Patient Symptoms and Quality of Life: A Randomized Phase II Trial. Int J Radiat Oncol Biol Phys. 2017;98:1036-44.

15. Cramer $\mathrm{H}$, Lauche $\mathrm{R}$, Klose $\mathrm{P}$, Lange $\mathrm{S}$, Langhorst J, Dobos GJ. Yoga for improving health-related quality of life, mental health and cancer-related symptoms in women diagnosed with breast cancer. Cochrane Database Syst Rev. 2017;2017. https://doi.org/10.1002/14651858.CD010802.pub2.

16. O'Neill M, Samaroo D, Lopez C, Tomlinson G, Santa Mina D, Sabiston C, et al. The Effect of Yoga Interventions on Cancer-Related Fatigue and Quality of Life for Women with Breast Cancer: A Systematic Review and Meta-Analysis of Randomized Controlled Trials. Integr Cancer Ther. 2020;19. https://doi.org/10.1177/ 1534735420959882.

17. Banerjee B, Vadiraj HS, Ram A, Rao R, Jayapal M, Gopinath KS, et al. Effects of an integrated yoga program in modulating psychological stress and radiationinduced genotoxic stress in breast cancer patients undergoing radiotherapy. Integr Cancer Ther. 2007;6:242-50.

18. Esper $P, M o F$, Chodak $G$, Sinner M, Cella D, Pienta KJ. Measuring quality of life in men with prostate cancer using the functional assessment of cancer therapyprostate instrument. Urology. 1997;50:920-8.

19. Baer RA, Smith GT, Hopkins J, Krietemeyer J, Toney L. Using self-report assessment methods to explore facets of mindfulness. Assessment. 2006;13:27-45

20. Yellen SB, Cella DF, Webster K, Blendowski C, Kaplan E. Measuring fatigue and other anemia-related symptoms with the Functional Assessment of Cancer Therapy (FACT) measurement system. J Pain Symptom Manag. 1997;13:63-74.

21. Szymanski KM, Wei JT, Dunn RL, Sanda MG. Development and validation of an abbreviated version of the expanded prostate cancer index composite instrument for measuring health-related quality of life among prostate cancer survivors. Urology. 2010;76:1245-50.

22. Cella D, Nichol MB, Eton D, Nelson JB, Mulani P. Estimating clinically meaningful changes for the functional assessment of cancer therapy - Prostate: Results from a clinical trial of patients with metastatic hormone-refractory prostate cancer. Value Heal. 2009;12:124-9.

23. Mukherjee N, Ji N, Hurez V, Curiel TJ, Montgomery MO, Braun AJ, et al. Intratumoral CD56bright natural killer cells are associated with improved survival in bladder cancer. Oncotarget. 2018:9:36492-502.

24. Skolarus TA, Dunn RL, Sanda MG, Chang P, Greenfield TK, Litwin MS, et al. Minimally important difference for the expanded prostate cancer index composite short form. Urology. 2015;85:101-6.

25. Nordin $\AA$, Taft C, Lundgren-Nilsson $\AA$, Dencker A. Minimal important differences for fatigue patient reported outcome measures-A systematic review. BMC Med Res Methodol. 2016;16. https://doi.org/10.1186/s12874-016-0167-6.

26. Park SW, Kim TN, Nam JK, Ha HK, Shin DG, Lee W, et al. Recovery of overall exercise ability, quality of life, and continence after 12-week combined exercise intervention in elderly patients who underwent radical prostatectomy: a randomized controlled study. Urology. 2012;80:299-306.

27. Vashistha V, Singh B, Kaur S, Prokop LJ, Kaushik D. The Effects of Exercise on Fatigue, Quality of Life, and Psychological Function for Men with Prostate Cancer: Systematic Review and Meta-analyses. Eur. Urol. Focus. 2016;2:284-95.

28. Lin PJ, Kleckner IR, Loh KP, Inglis JE, Peppone LJ, Janelsins MC, et al. Influence of Yoga on Cancer-Related Fatigue and on Mediational Relationships Between Changes in Sleep and Cancer-Related Fatigue: A Nationwide, Multicenter Randomized Controlled Trial of Yoga in Cancer Survivors. Integr Cancer Ther 2019;18. https://doi.org/10.1177/1534735419855134.

29. Lyman GH, Greenlee H, Bohlke K, Bao T, DeMichele AM, Deng GE, et al. Integrative therapies during and after breast cancer treatment: ASCO endorsement of the SIO clinical practice guideline. J Clin Oncol. 2018;36:2647-55.

30. Glaser R, Kiecolt-Glaser JK. Stress-induced immune dysfunction: implications for health. Nat Rev Immunol. 2005;5:243-51.

31. Miller AH, Spencer RL, Pearce BD, Pisell TL, Azrieli $Y$, Tanapat $P$, et al. Glucocorticoid receptors are differentially expressed in the cells and tissues of the immune system. Cell Immunol. 1998;186:45-54.

32. Fan KQ, Li YY, Wang HL, Mao XT, Guo JX, Wang F, et al. Stress-induced metabolic disorder in peripheral CD4+ $\mathrm{T}$ cells leads to anxiety-like behavior. Cell. 2019;179:864-.e19.
33. Bower JE, Ganz PA, May LT, Hu W, Belin TR, Sepah S, et al. Inflammatory biomarkers and fatigue during radiation therapy for breast and prostate cancer. Clin Cancer Res. 2009;15:5534-40.

34. Bussolino F, Wang JM, Defilippi P, Turrini F, Sanavio F, Edgell CJS, et al. Granulocyte- and granulocyte- macrophage-colony stimulating factors induce human endothelial cells to migrate and proliferate. Nature. 1989:337:471-3.

35. Santaella A, Kuiperij HB, van Rumund A, Esselink RAJ, van Gool AJ, Bloem BR, et al. Cerebrospinal fluid monocyte chemoattractant protein 1 correlates with progression of Parkinson's disease. npj Park Dis. 2020;6. https://doi.org/10.1038/ s41531-020-00124-z.

36. Lee WJ, Liao YC, Wang YF, Lin IF, Wang SJ, Fuh JL. Plasma MCP-1 and cognitive decline in patients with Alzheimer's disease and mild cognitive impairment: A two-year follow-up study. Sci Rep. 2018;8. https://doi.org/10.1038/s41598-01819807-y.

37. Lu Y, Chen Q, Corey E, Xie W, Fan J, Mizokami A, et al. Activation of MCP-1/CCR2 axis promotes prostate cancer growth in bone. Clin Exp Metastasis. 2009;26:161-9.

38. Ramos MI, Tak PP, Lebre MC. Fms-like tyrosine kinase 3 ligand-dependent dendritic cells in autoimmune inflammation. Autoimmun Rev. 2014;13:117-24.

39. Bower JE, Garet D, Sternlieb B, Ganz PA, Irwin MR, Olmstead R, et al. Yoga for persistent fatigue in breast cancer survivors: a randomized controlled trial. Cancer. 2012;118:3766-75.

40. Zahavich ANR, Robinson JA, Paskevich D, Culos-Reed SN. Examining a therapeutic yoga program for prostate cancer survivors. Integr Cancer Ther. 2013;12:113-25.

41. Sadja J, Mills PJ. Effects of yoga interventions on fatigue in cancer patients and survivors: a systematic review of randomized controlled trials. Explor J Sci Heal. 2013;9:232-43.

\section{ACKNOWLEDGEMENTS}

The manuscript was copy-edited by Ms. Susan Albrecht at MedSurgBio Ltd. This work was supported by: (1) the ThriveWell Cancer Foundation (Dharam Kaushik); (2) P30 Cancer Center Support Grant from the National Cancer Institute (CA054174) (Mays Cancer Center at University of Texas Health San Antonio) (Dharam Kaushik) (3) the Roger L. And Laura D. Zeller Charitable Foundation Chair in Urologic Cancer (Robert S. Svatek), (4) the Glenda and Gary Woods Distinguished Chair in GU Oncology (Michael A. Liss), (5) Department of Defense, Prostate Cancer Research Program (PCRP) Physician Research Training Award. Office of the Assistant Secretary of Defense for Health Affairs through the Prostate Cancer Research Program under Award No. W81XWH-15-1-0441 (Michael A. Liss) (6) a Research Training Award (RP170345) from the Cancer Prevention \& Research Institute of Texas (Furkan Dursun) (7) Stanley and Sandra Rosenberg Endowed Chair in Urologic Research (Dharam Kaushik) The funding sources had no role in the design and conduct of the study; collection, management, analysis, and interpretation of the data; preparation, review, or approval of the manuscript; and decision to submit the manuscript for publication

\section{AUTHOR CONTRIBUTIONS}

DK conceived and designed the study. DK, PS, RSS, and MAL acquired, analyzed, or interpreted the data. DK drafted the manuscript. DK and PS verified the data. PS and HW performed the statistical analysis. DK, RSS, and MAL provided administrative, technical, or material support and obtained funding. DK supervised the study. All authors critically revised the manuscript for important intellectual content.

\section{COMPETING INTERESTS}

The authors declare no competing interests.

\section{ADDITIONAL INFORMATION}

Supplementary information The online version contains supplementary material available at https://doi.org/10.1038/s41391-021-00470-w.

Correspondence and requests for materials should be addressed to Dharam Kaushik.

Reprints and permission information is available at http://www.nature.com/ reprints

Publisher's note Springer Nature remains neutral with regard to jurisdictional claims in published maps and institutional affiliations. 
Open Access This article is licensed under a Creative Commons Attribution 4.0 International License, which permits use, sharing, adaptation, distribution and reproduction in any medium or format, as long as you give appropriate credit to the original author(s) and the source, provide a link to the Creative Commons license, and indicate if changes were made. The images or other third party material in this article are included in the article's Creative Commons license, unless indicated otherwise in a credit line to the material. If material is not included in the article's Creative Commons license and your intended use is not permitted by statutory regulation or exceeds the permitted use, you will need to obtain permission directly from the copyright holder. To view a copy of this license, visit http://creativecommons. org/licenses/by/4.0/.

(c) The Author(s) 2021 